\title{
Viabilidade do Hidrogel na Recuperação de Cerrado sensu stricto com Espécies Nativas
}

\author{
Lauriane Fonseca ${ }^{1}$, Iris Roitman ${ }^{2}$, Tamiel Khan Baiocchi Jacobson ${ }^{2}$, \\ Roberto Shojirou Ogata ${ }^{3}$, Raphael Alberto Fhur Solari², \\ Rômulo José da Costa Ribeiro ${ }^{2}$
}

\author{
${ }^{1}$ Faculdade de Planaltina - FUP, Universidade de Brasília - UnB, Planaltina/DF, Brasil \\ ${ }^{2}$ Projeto Radis, Faculdade de Planaltina - FUP, Universidade de Brasília - UnB, Planaltina/DF, Brasil \\ ${ }^{3}$ Viveiro Cerrado Vivo, Planaltina/DF, Brasil
}

\begin{abstract}
RESUMO
Este estudo determinou o custo e o efeito de polímero hidrorretentor (hidrogel) na redução da mortandade de mudas de seis espécies florestais nativas do Cerrado, na recuperação de área degradada em Planaltina - Distrito Federal. Foram testados quatro tratamentos (com e sem hidrogel nas estações seca e chuvosa) em delineamento em blocos casualizados com quatro blocos e três repetições por bloco. ANOVA e teste de Tukey revelaram que o hidrogel reduziu significativamente a mortandade na estação seca, mas não teve efeito significativo na estação chuvosa. Considerando o custo de substituição de mudas mortas, o tratamento sem hidrogel foi mais caro que com hidrogel na estação seca. O plantio no final da estação seca com hidrogel representa alternativa viável para reduzir a mortandade de mudas via mitigação dos efeitos do estresse hídrico pós-transplante no final da estação seca, sem representar custo significativo adicional aos programas de recuperação de áreas degradadas de Cerrado.
\end{abstract}

Palavras-chave: polímero hidrorretentor, recuperação de áreas degradadas, cerrado sensu stricto.

\section{Viability of Hydrogel in cerrado sensu stricto Revegetation with Native Species}

\begin{abstract}
We determined the cost and effect of a water retention polymer (hydrogel) in reducing mortality of planted seedlings of six Cerrado forest species in the restoration of a degraded area in Planaltina county - Brazilian Federal District. We tested four treatments (hydrogel use and no hydrogel use in dry and rainy seasons) in a randomized block design, with four blocks and three replications per block. ANOVA and Tukey's test revealed that hydrogel significantly reduced mortality in the dry season, and had no significant effect in the rainy season. Regarding the cost of dead seedlings replacement, the treatment without hydrogel was more expensive than the one with hydrogel in the dry season. Planting at the end of the dry season using hydrogel is a viable alternative in reducing mortality of seedlings via mitigation of water stress effects after transplantation, without significant additional cost to recovery programs of Cerrado degraded areas.
\end{abstract}

Keywords: water retention polymer, ecological restoration, cerrado sensu stricto. 


\section{INTRODUÇÃO}

O Cerrado vem sofrendo com significativas alterações antrópicas da paisagem natural. Nos últimos 35 anos, mais da metade dos dois milhões de $\mathrm{km}^{2}$ da paisagem natural do bioma já foi transformada em pastagens, monocultura, áreas para mineração e outros usos. Em 2005, 55\% do Cerrado já havia sido desmatado, o que representa três vezes a área desmatada da floresta Amazônica (Klink \& Machado, 2005). Essas alterações no uso do solo resultaram em extensas áreas antropizadas ou com alto grau de degradação (Sano et al., 2008). Segundo Corrêa (1998), áreas degradadas podem ser definidas como ecossistemas naturais alterados, tanto em sua estrutura como em suas funções ecossistêmicas. Os objetivos da recuperação de áreas degradadas são restaurar a estrutura e recuperar as funções do ecossistema, seja na produção de alimentos e matérias-primas, ou na prestação de serviços ecossistêmicos (Rodrigues \& Gandolfi, 2001; Engel \& Parrota, 2003; Aquino et al., 2009).

Uma das principais causas de mortalidade e baixo desenvolvimento de mudas em plantios para recuperação de áreas degradadas do Cerrado é o estresse hídrico, devido ao clima sazonal, com precipitações entre 800 e $1.800 \mathrm{~mm}$ anuais, das quais $90 \%$ precipitam entre outubro e abril (Bustamante et al., 2012). A falta de retenção hídrica dos substratos nos meses secos pós-semeadura tem sido o principal fator do fracasso em projetos de revegetação no Cerrado (Corrêa \& Cardoso, 1998). O plantio de mudas em programas de recuperação de áreas degradadas no Cerrado geralmente é realizado durante o período chuvoso. Esse período é mais propício ao desenvolvimento de mudas plantadas e também de plantas espontâneas competidoras. Por outro lado, a garantia da disponibilidade hídrica de forma localizada por meio de irrigação e/ou uso de polímeros hidrorretentores em plantios de mudas no final da estação seca pode fornecer vantagem competitiva inicial para o estrato arbóreo em relação ao herbáceo, aumentando assim a probabilidade do estabelecimento das mudas no período chuvoso. A utilização de polímeros hidrorretentores (também chamado de hidrogel, polímero hidroabsorvente e polímero superabsorvente) na agricultura teve início na década de 1950, com seus efeitos benéficos reconhecidos desde então, no que diz respeito ao aumento da retenção de água no solo, redução da lixiviação de nutrientes, melhoria na capacidade de troca catiônica e maior disponibilidade de água para as plantas (Azevedo et al., 2002; Ekebafe et al., 2011; Bakass et al., 2002).

Contudo, ainda não há consenso quanto à efetividade do hidrogel sobre a sobrevivência e desenvolvimento de plantas. Alguns estudos indicam que enquanto o hidrogel pode favorecer o estabelecimento de mudas no campo, a aplicação de grandes quantidades de hidrogel pode ter efeito negativo sobre seu desenvolvimento (Sarvaš, 2003; Sarvaš et al., 2007). Estudos verificaram que o hidrogel favoreceu a retenção de água no solo (Oliveira et al., 2004) e teve efeito positivo no desenvolvimento de espécies como o eucalipto (Bernardi et al., 2012; Thomas, 2008), café (Azevedo et al., 2002), pinhão-manso (Dranski et al., 2013) e amora (Moreira et al., 2011) e ipê amarelo (Mews et al., 2015). Entretanto, outros estudos apontam para outra direção. Sousa et al. (2013) constataram não haver efeito positivo do hidrogel sobre a sobrevivência e crescimento de mudas de angico vermelho em viveiro e encontraram efeito negativo sobre o desenvolvimento radicular (massa seca das raízes) com o aumento da dosagem do hidrogel. Também não houve efeito significativo do uso do hidrogel na sobrevivência de mudas no campo para trinta espécies nativas da mata Atlântica (Barbosa et al. 2013). A utilização do hidrogel para a produção de mudas de espécies lenhosas nativas do Cerrado em plantios de recuperação ainda é pouco estudada (Venturoli et al., 2013; Monteiro, 2014). Outra lacuna a respeito do uso do hidrogel é a falta da avaliação do custo-benefício de sua utilização em programas de recuperação de áreas degradadas no Cerrado. Venturoli et al. (2013) e Monteiro (2014) verificaram inexistência do efeito do hidrogel na sobrevivência e desenvolvimento de onze espécies arbóreas nativas do cerrado, plantadas na estação chuvosa, na recuperação de área degradada no Cerrado. O plantio de mudas na recuperação de áreas degradadas durante o final da estação seca (antes da estação chuvosa) pode favorecer o desenvolvimento de mudas plantadas, que terão maior capacidade de enfrentar a competição contra espécies espontâneas que germinam no período chuvoso.

\section{OBJETIVOS}

O presente estudo pretende verificar: a) se existe efeito significativo do uso do hidrogel na sobrevivência de mudas de seis espécies florestais nativas do Cerrado 
até trinta dias após o plantio, na recuperação de área degradada no Cerrado durante os períodos seco e chuvoso; e b) se o custo de utilização do hidrogel na seca é compensado pela diminuição no custo de substituição das mudas não estabelecidas. A hipótese de estudo é de que o uso do hidrogel no plantio de mudas durante a estação seca tem efeito significativo sobre a redução da mortandade, compensando o seu custo de utilização. Além disso, espera-se que o uso do hidrogel em plantio realizado durante a estação chuvosa não apresente efeito significativo sobre a mortandade.

\section{MATERIAL E MÉTODOS}

\section{1. Área de estudo}

A área de estudo localiza-se em Planaltina, Distrito Federal (15 40’19,75" S; 47 38'25,08”; altitude $943 \mathrm{~m}$ ) (Figura 1). O experimento foi realizado em área de pastagem degradada, (Urochloa brizantha) (Hochst. ex A.Rich.) R.Webster, anteriormente ocupada por cerrado sensu stricto. A área é homogênea em relação à topografia e declividade. O solo é classificado como Latossolo Vermelho (EMBRAPA, 2006), álico, levemente ácido e com textura argilosa. O clima é do tipo Aw (classificação de Köppen), com duas estações bem definidas: seca e chuvosa, com veranicos durante a estação das chuvas. A precipitação média anual varia em torno $1.500 \mathrm{~mm}$ e a temperatura média anual varia entre 21,3 e $27,2^{\circ} \mathrm{C}$ (Malaquias et al., 2010).

\subsection{Delineamento experimental}

O delineamento experimental consistiu em blocos casualizados com quatro blocos (paralelos e com espaçamento de $5 \mathrm{~m}$ entre si), três repetições por bloco e quatro tratamentos: plantio na estação seca com hidrogel $\left(\mathrm{t}_{1}\right)$, plantio na estação seca sem hidrogel $\left(\mathrm{t}_{2}\right)$, plantio na estação chuvosa sem hidrogel $\left(\mathrm{t}_{3}\right)$, e plantio na estação chuvosa com hidrogel $\left(\mathrm{t}_{4}\right)$. Cada unidade amostral (com dimensões de $3 \mathrm{~m} \times 3 \mathrm{~m}$ ) foi composta por

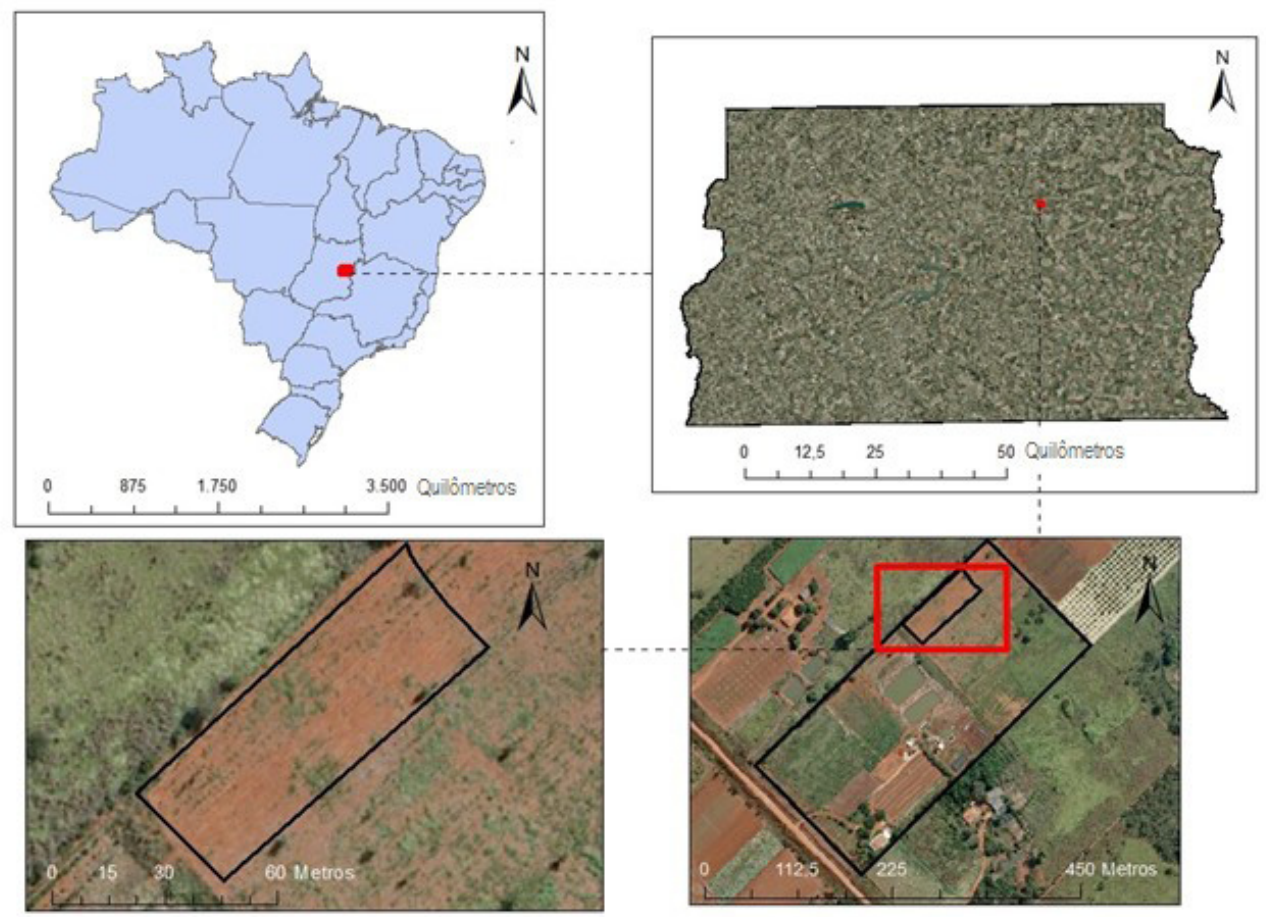

Figura 1. Localização da área de estudo em Planaltina, Distrito Federal, e fotografia aérea da área do experimento em 2013 na propriedade rural, disponibilizada pela Companhia de Planejamento do Distrito Federal (CODEPLAN). Figure 1. Location of the study area in Planaltina, Brazilian Federal District, and aerial photograph of the experimental area in 2013 in the rural property provided by Federal District's Planning Company (CODEPLAN). 
doze mudas, em disposição de quincôncio, conforme Kageyama \& Gandara (2000), com espaçamento das mudas de $1 \times 1 \mathrm{~m}$. Para manter a mesma densidade de plantas dentro e entre unidades amostrais, foram adicionadas duas mudas na borda e uma no centro de cada parcela. Os dados dessas mudas não foram utilizados no experimento para que se mantivesse um número igual de mudas por espécie em cada parcela (Figura 2).

Foram utilizadas mudas de mesma idade (um ano) de seis espécies florestais nativas do Cerrado: Tapirira guianensis Aubl., Genipa americana L., Cedrela fissilis Vell., Hymeneae courbaril L., Handroanthus serratifolius (A. H. Gentry) S. Grose e Inga cylindrica (Vell.) Mart., com duas mudas por espécie, distribuídas aleatoriamente em cada parcela. Espécies de mata ripária (matas de galeria e ciliar) são utilizadas em projetos de recuperação de áreas de cerrado sensu stricto. A razão para isso é que apresentam crescimento inicial mais rápido que as espécies dessa fitofisionomia e porque recobrem o solo com maior rapidez, reduzindo o efeito da competição com gramíneas exóticas (Felfili et al., 2005; Felfili, 2007).

Em todas as covas (com dimensões de $0,3 \times 0,3 \times 0,3 \mathrm{~m}$ ), foram adicionados $200 \mathrm{~g}$ de fonte de matéria orgânica (esterco bovino), $50 \mathrm{~g}$ de uma fonte química de micronutrientes (17\% Ca, $8 \% \mathrm{Mg}, 5 \% \mathrm{~S}, 0,3 \% \mathrm{~B}, 1 \% \mathrm{Zn}$ ), e 50g de NPK - 4:14:18. Nos tratamentos com hidrogel foram adicionados $1.000 \mathrm{ml}$ de hidrogel hidratado ( $5 \mathrm{~g}$ de massa seca) por cova ( $25 \mathrm{~g} / \mathrm{kg}$ de solo). O plantio dos tratamentos $t_{1}$ e $t_{2}$ foi realizado em setembro de 2013 (final da estação seca), enquanto o plantio dos tratamentos $\mathrm{t}_{3} \mathrm{et}_{4}$ foi realizado em novembro do mesmo ano (estação chuvosa) (Figura 3). A verificação da mortandade foi realizada trinta dias após o plantio.

\subsection{Análise estatística dos dados}

A normalidade dos dados da variável mortandade (número de indivíduos mortos por parcela) foi testada por meio do teste d'Agostino $(\alpha=0,05)$, adequado para amostras pequenas $(\mathrm{N}>10)$ com o software BioEstat $^{\circledR}$ (Ayres et al., 2007). Para verificar se houve diferença significativa na mortandade de mudas entre tratamentos, foi realizada a análise variância (ANOVA) para $\alpha=0,05$ e $\alpha=0,01$. Para verificar se houve diferença significativa entre pares de tratamentos, foi realizado o teste de comparação de médias de Tukey (ao nível de 5\% de probabilidade) conforme Ferreira (1996).

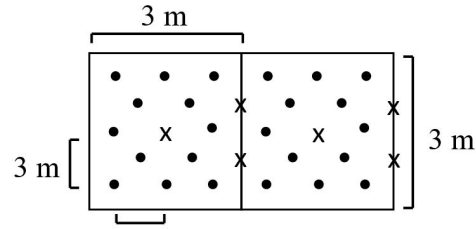

- mudas plantadas incluídas na análise de dados

$\mathrm{x}$ mudas plantadas excluídas da análise de dados

Figura 2. Disposição e espaçamento $(1 \mathrm{~m} \times 1 \mathrm{~m})$ das mudas plantadas em duas unidades amostrais $(3 \mathrm{~m} \times 3 \mathrm{~m})$ contíguas.

Figure 2. Position and spacing $(1 \mathrm{~m} \times 1 \mathrm{~m})$ of seedling in two contiguous sampling units $(3 \mathrm{~m} \times 3 \mathrm{~m})$.

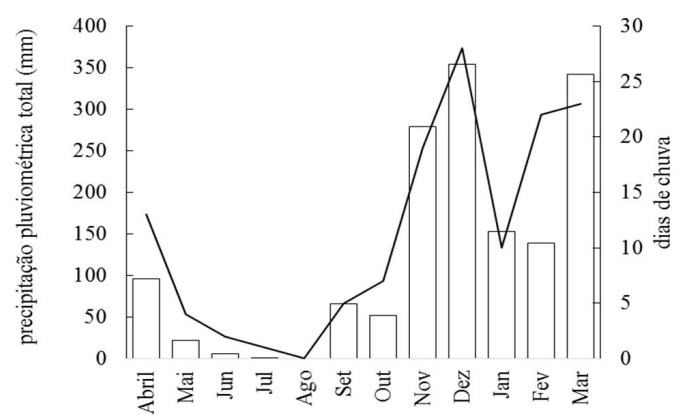

Figura 3. Precipitação pluviométrica total mensal (barras) e dias de chuva (linha) entre abril de 2013 e março de 2014, registrados na estação climatológica mais próxima da área de estudo (estação Brasília). Fonte: Instituto Nacional de Meteorologia - INMET (2013).

Figure 3. Total monthly pluviometric precipitation (bars) and days of rain (line) between April, 2013 and March, 2014, registered at the closest climatological station to the study area (Brasília station). Source: Instituto Nacional de Meteorologia - INMET (2013).

Foram determinados os custos (insumos e mão de obra) da execução do plano de recuperação. O custo de substituição de mudas mortas foi comparado ao custo adicional do uso de hidrogel.

\section{RESULTADOS E DISCUSSÃO}

Os dados apresentaram distribuição normal segundo o teste de D’ Agostino. A análise de variância acusou diferença significativa entre tratamentos (Tabela 1). A maior mortandade ocorreu no tratamento $t_{2}$, que foi significativamente diferente dos demais tratamentos (Tabela 2). 
Tabela 1. Análise de variância para comparação dos quatro tratamentos testados. Onde GL = grau de liberdade; $\mathrm{SQ}=$ soma dos quadrados; $\mathrm{QM}=$ quadrado médio.

Table 1. Analysis of variance comparing four treatments. Where GL = degrees of freedom; $S Q$ = sum of squares; $\mathrm{QM}=$ mean square.

\begin{tabular}{lcccccc} 
Fontes de variação & $\mathbf{G L}$ & $\mathbf{S Q}$ & $\mathbf{Q M}$ & $\mathbf{F}_{\text {calculado }}$ & $\begin{array}{c}\mathbf{F}_{\text {tabelado }} \\
(\mathbf{a}=\mathbf{0 . 0 5})\end{array}$ & $\begin{array}{c}\mathbf{F}_{\text {tabelado }} \\
(\mathbf{a}=\mathbf{0 . 0 1})\end{array}$ \\
Tratamentos & 3 & 59,23 & 19,74 & 8,89 & 3,86 & 6,99 \\
Blocos & 3 & 26,73 & 8,91 & & & \\
Resíduo & 41 & 91,02 & 2,22 & & \\
Total & 48 & 176,98 & & & \\
\hline
\end{tabular}

Tabela 2. Mortandade total e mortandade média por tratamento de mudas e teste de comparação de médias de Tukey $\left(\Delta_{\text {crítico }}=1,63\right)$. Os tratamentos com a mesma letra não diferem entre si.

Table 2. Total and mean seedling mortality per treatment and Tukey's test for comparison of means $\left(\Delta_{\text {critical }}=1.63\right)$. Treatments with the same letter are similar.

\begin{tabular}{cccc} 
Tratamento & Mortandade total & Mortandade média & Desvio padrão \\
\hline$t_{2}$ & 75 & $6,25 \mathrm{a}$ & 1,44 \\
$\mathrm{t}_{1}$ & 47 & $3,92 \mathrm{~b}$ & 1,76 \\
$\mathrm{t}_{3}$ & 46 & $3,83 \mathrm{~b}$ & 2,17 \\
$\mathrm{t}_{4}$ & 41 & $3,42 \mathrm{~b}$ & 0,90 \\
\hline
\end{tabular}

Os resultados confirmaram a hipótese de que o uso do hidrogel na estação seca leva à redução na mortandade de indivíduos e não tem efeito significativo na redução da mortandade na estação chuvosa. Isto pode ser devido ao aumento da capacidade de retenção de água no solo, atenuando o estresse inicial pós-transplante. O transplante no final da estação seca expõe as mudas a períodos de baixa umidade, quando o potencial hídrico da atmosfera é baixo, intensificando a perda de umidade da membrana radicular. A desidratação dos tecidos é causada pelo desbalanço entre a absorção de água pelas raízes e a transpiração foliar. Sob condições de estresse hídrico, a regulação da absorção de água pelas raízes é mais importante que a regulação da transpiração foliar para a superação do estresse hídrico (Aroca et al., 2012). O aumento no tempo de absorção de água no solo está ligado à capacidade do hidrogel em utilizar tanto a água gravitacional como a água capilar do solo. Um estudo que avaliou a retenção de água no solo com hidrogel revelou que, tanto em solos de textura arenosa como argilosa, o uso do hidrogel aumentou o conteúdo de água residual e saturada, no entanto, o aumento na saturação foi maior em solos arenosos (Abedi-Koupai et al., 2008). No caso do presente estudo (solo argiloso), o hidrogel possivelmente atuou como atenuante do estresse hídrico durante o período seco em
Latossolo Vermelho. Diminuir o estresse pós-plantio, favorece competitividade por luz, água e nutrientes durante o período chuvoso, em detrimento de plantas espontâneas, diminuindo assim a mortandade. Esse favorecimento pode representar uma variável importante para o sucesso das estratégias de recuperação de áreas degradadas no Cerrado com esse tipo de solo. Segundo Apostol et al. (2009), o período pós-transplante representa o estágio mais crítico de dissecação de mudas no campo. Os autores constataram que o uso do hidrogel diminuiu o ressecamento da membrana radicular em mudas pré-transplantadas de Quercus rubra L. e que mudas tratadas com hidrogel apresentaram umidade $80 \%$ superior em relação a mudas não tratadas com esse polímero. Sob condições de estresse hídrico, o uso do hidrogel diminui o número médio de dias para o início da brotação, combinado com o atraso da perda de umidade dos tecidos radiculares, aliviando o estresse inicial pós-transplante, aumentando a sobrevivência em curto prazo.

No presente estudo, o plantio das mudas sem hidrogel na estação seca apresentou mortandade de $41 \%$, enquanto a mortandade com o uso de hidrogel foi de $26 \%$. A efetividade do hidrogel na melhoria do desenvolvimento e na redução da mortandade de mudas depende de vários fatores como a espécie botânica 
Tabela 3. Orçamento detalhado dos custos de plantio para tratamentos com a aplicação de hidrogel ( $t_{1}$ e $\left.t_{3}\right)$ e sem aplicação de hidrogel ( $\mathrm{t}_{2}$ e $\left.\mathrm{t}_{4}\right)$.

Table 3. Detailed budget for planting for treatments with $\left(t_{1}\right.$ and $\left.t_{3}\right)$ and without hydrogel $\left(t_{2}\right.$ and $\left.t_{4}\right)$.

\begin{tabular}{|c|c|c|c|c|c|}
\hline Descrição & $\begin{array}{l}\text { Quantidade } \\
\text { por muda }\end{array}$ & $\begin{array}{l}\text { Quantidade por } \\
\text { tratamento }\end{array}$ & Preço & $\begin{array}{l}\text { Custo por } \\
\text { muda (R\$) }\end{array}$ & $\begin{array}{l}\text { Custo por } \\
\text { tratamento } \\
(\mathrm{R} \$)\end{array}$ \\
\hline Mudas: & 1 muda & 144 mudas & $\mathrm{R} \$ 4,00 /$ muda & 4,00 & 576,00 \\
\hline Fertilizante orgânico & $200 \mathrm{~g}$ & $28.800 \mathrm{~g}$ & $\mathrm{R} \$ 0,70 / \mathrm{kg}$ & 0,14 & 20,16 \\
\hline $\begin{array}{l}\text { Fertilizante químico com } \\
\text { micronutrientes }\end{array}$ & $50 \mathrm{~g}$ & $7.200 \mathrm{~g}$ & $\mathrm{R} \$ 11,50 / \mathrm{kg}$ & 0,58 & 82,80 \\
\hline Adubo químico $\mathrm{N}_{4} \mathrm{P}_{14} \mathrm{~K}_{8}$ & $50 \mathrm{~g}$ & $7.200 \mathrm{~g}$ & $\mathrm{R} \$ 1,59 / \mathrm{kg}$ & 0,08 & 11,45 \\
\hline Isca formicida & 0,0069 dias & $1 \mathrm{~kg}$ & $\mathrm{R} \$ 6,00 / \mathrm{kg}$ & 0,04 & 6,00 \\
\hline Mão de obra & 0,042 dias & 15 diárias & $\mathrm{R} \$ 40,00 /$ dia & 4,17 & 600,00 \\
\hline Hidrogel (5g/litro; 1 l/cova) & $5 \mathrm{~g}$ & $720 \mathrm{~g}$ & $\mathrm{R} \$ 9,30 / 250 \mathrm{~g}$ & 0,19 & 26,78 \\
\hline $\begin{array}{l}\text { Valor total (tratamento } \\
\text { sem hidrogel) }\end{array}$ & & & & 9,00 & $1.296,41$ \\
\hline $\begin{array}{l}\text { Valor total (tratamento } \\
\text { com hidrogel) }\end{array}$ & & & & 9,19 & $1.323,19$ \\
\hline
\end{tabular}

Tabela 4. Custo total dos tratamentos incluindo o custo de substituição de mudas (replantio) devido à mortandade. Table 4. Total cost of each treatment including seedling replacement due to mortality.

\begin{tabular}{cccc} 
Tratamento & $\begin{array}{c}\text { Número de mudas } \\
\text { replantadas }\end{array}$ & $\begin{array}{c}\text { Custo do replantio } \\
(\mathbf{R} \$)\end{array}$ & $\begin{array}{c}\text { Custo total } \\
\text { (Plantio + replantio) (R\$) }\end{array}$ \\
\hline$t_{1}$ & 47 & 431,93 & 1755,12 \\
$t_{2}$ & 75 & 675,00 & 1971,41 \\
$t_{3}$ & 46 & 422,74 & 1745,93 \\
$t_{4}$ & 41 & 369,00 & 1665,41 \\
\hline
\end{tabular}

utilizada, a quantidade de hidrogel aplicada, o tipo de solo e frequência de irrigação ou precipitação (Kazanskii \& Dubrovskii, 1992; Mangold \& Sheley, 2007). Outros estudos não apontam o hidrogel como responsável pela diminuição da mortandade de mudas na recuperação de áreas degradadas no Cerrado. A elevada intensidade de chuvas após o plantio pode contribuir para a alta percolação do hidrogel no solo, o que também pode interferir em resultados de baixa efetividade dos efeitos do hidrogel no solo (Venturoli et al., 2013; Monteiro, 2014). Chirino et al. (2011) verificaram que a associação de hidrogel, argila e turfa reduziu o estresse hídrico no estabelecimento de mudas de Quercus suber L. no campo e pode contribuir para melhorar métodos de restauração florestal em ecossistemas semiáridos.

Considerando os custos do projeto de recuperação, o custo total por muda plantada com o uso de hidrogel foi de $R \$ 9,19$, enquanto o custo total por muda sem o uso do hidrogel foi de $\mathrm{R} \$$ 9,00 (diferença de $\mathrm{R}$ \$ 0,19/muda) (Tabela 3). Ao considerar o custo do replantio de mudas devido à mortandade, $\mathrm{o}$ tratamento $\mathrm{t}_{2}$ apresentou maior custo total (plantio + replantio $=\mathrm{R} \$ 1.971,62$ ) (Tabela 4), valor este superior em R $\$ 216,55$ ( $\mathrm{R} \$ 269,85)$ em relação ao custo total do tratamento $t_{1}$.

\section{CONCLUSÃO}

O custo total do plantio de mudas com uso do hidrogel no final da estação seca foi menor que o custo total do plantio de mudas sem hidrogel na mesa época. Os custos com substituição de mudas no tratamento sem hidrogel superaram os custos da utilização do hidrogel na estação seca. Porém, se o plantio for realizado na estação chuvosa, o uso do hidrogel torna-se dispensável. O uso de hidrogel pode ser uma boa alternativa para plantios de mudas em estratégias de recuperação de áreas degradadas do Cerrado, sem aumentar significativamente o custo total do plantio. 


\section{AGRADECIMENTOS}

Agradecemos ao Viveiro Cerrado Vivo e o apoio financeiro concedido pelo Projeto Regularização Ambiental e Diagnóstico dos Sistemas Agrários dos Assentamentos da Região Norte do Estado do Mato Grosso - Radis (Projeto 5788 - FUB/FUP, Sub-Processo: 14018/2015) e pelo CNPq (Processo: 161329/2013-6).

\section{STATUS DA SUBMISSÃO}

Recebido: 21 nov., 2016

Aceito: 22 dez., 2016

\section{AUTOR(ES) PARA CORRESPONDÊNCIA}

\section{Iris Roitman}

Faculdade de Planaltina, Universidade de Brasília - UnB, Área Universitária 1, Vila Nossa Senhora de Fátima, CEP 73340-710, Planaltina, DF, Brasil e-mail: irisroitman01@gmail.com

\section{REFERÊNCIAS}

Abedi-Koupai J, Sohrab F, Swarbrick G. Evaluation of hydrogel application on soil water retention characteristics. Journal of Plant Nutrition 2008; 31(2): 317-331. http:// dx.doi.org/10.1080/01904160701853928.

Apostol KG, Jacobs DF, Dumroese RK. Root desiccation and drought stress responses of bareroot Quercus rubra seedlings treated with a hydrophilic polymer root dip. Plant and Soil 2009; 315(1-2): 229-240. http://dx.doi. org/10.1007/s11104-008-9746-6.

Aquino FG, Oliveira MC, Ribeiro JF, Passos FB. Módulos para recuperação de Cerrado com espécies nativas de uso múltiplo. Planaltina: Embrapa Cerrados; 2009.

Aroca R, Porcel R, Ruiz-Lozano JM. Regulation of root water uptake under abiotic stress conditions. Journal of Experimental Botany 2012; 63(1): 43-57. PMid:21914658. http://dx.doi.org/10.1093/jxb/err266.

Ayres M, Ayres M Jr, Ayres DL, Santos AL. BIOESTAT 5.0: aplicações estatísticas nas áreas das Ciências Biológicas e da Saúde. Mamirauá: IDSM/MCT/CNPq; 2007.

Azevedo TLF, Bertonha A, Gonçalves ACA, Freitas PSL, Rezende L, Frizzone JA. Níveis de polímero superabsorvente, frequências de irrigação e crescimento de mudas de café. Acta Scientiarum 2002; 5(24):1239-1243.

Bakass M, Mokhlisse A, Lallemant M. Absorption and desorption of liquid water by a superabsorbent polymer: effect of polymer in the drying of the soil and the quality of certain plants. Journal of Applied Polymer Science 2002; 83(2): 234-24. http://dx.doi.org/10.1002/app.2239.

Barbosa TC, Rodrigues RR, Couto HTZ. Tamanhos de recipientes e o uso de hidrogel no estabelecimento de mudas de espécies florestais nativas. Hoehnea 2013; 40(3): 537556. http://dx.doi.org/10.1590/S2236-89062013000300013.

Bernardi MR, Sperotto MS Jr, Daniel O, Vitorino ACT. Crescimento de mudas de Corymbia citriodora em função do uso de hidrogel e adubação. Cerne 2012; 18(1): 67-74. http://dx.doi.org/10.1590/S0104-77602012000100009.

Bustamante MMC, Nardoto GB, Pinto AS, Resende JCF, Takahashi FSC, Vieira LCG. Pontential impacts of climate change on biogeochemical functioning of Cerrado ecosystems. Brazilian Journal of Biology = Revista Brasileira de Biologia 2012; 72(3): 65-671. http://dx.doi.org/10.1590/ S1519-69842012000400005.

Chirino E, Vilagrosa A, Vallejo VR. Using hydrogel and clay to improve the water status of seedlings for dryland restoration. Plant and Soil 2011;344(1-2): 99-110. http:// dx.doi.org/10.1007/s11104-011-0730-1.

Corrêa RS. Degradação e recuperação de áreas no Distrito Federal. In: Corrêa RS, Melo B Fo, editores. Ecologia e recuperação de áreas degradadas no cerrado. Brasília: Paralelo 15; 1998.

Corrêa RS, Cardoso ES. Espécies testadas na revegetação de áreas degradadas. In: Corrêa RS, Melo B Fo, editores. Ecologia e recuperação de áreas degradadas no cerrado. Brasília: Paralelo 15; 1998.

Dranski JAL, Pinto AS Jr, Campagnolo MA, Malavasi UC, Malavasi MM, Guimarães VF. Sobrevivência e crescimento inicial de pinhão-manso em função da época de plantio e do uso de hidrogel. Ciência Florestal 2013; 23(3): 489-498. http://dx.doi.org/10.5902/1980509810560.

Ekebafe LO, Ogbeifun DE, Okieimen FE. Polymer applications in agriculture. Biokemistri 2011; 23(2): 81-89.

Empresa Brasileira de Pesquisa Agropecuária - EMBRAPA. Sistema brasileiro de classificação de solos. 2. ed. Rio de Janeiro: EMBRAPA-SPI; 2006.

Engel VL, Parrota JA. Definindo a restauração ecológica: tendências e perspectivas mundiais. In: Kageyama PY, Oliveira RE, Moraes LFD, Engel VL, Ganrada FB, editores. Restauração ecológica de ecossistemas naturais. Botucatu: Fundação de Estudos e Pesquisas Agrícolas e Florestais; 2003.

Felfili JM. Recuperação de áreas degradadas no Cerrado, com espécies nativas do Bioma: quebrando paradigmas. Revista Opiniões 2007; 7.

Felfili JM, Fagg CW, Pinto JRR. Modelo nativas do bioma: stepping stones na formação de corredores ecológicos, pela recuperação de áreas degradadas no cerrado. In: Arruda $\mathrm{MB}$, editor. Gestão Integrada de Ecossistemas Aplicada a Corredores Ecológicos. Brasília: Ibama; 2005. 
Ferreira PV. Estatística experimental aplicada à Agronomia. 2. ed. Maceió: EDUFAL; 1996.

Instituto Nacional de Meteorologia - INMET. Dados meteorológicos. 2013 [citado em 2013 dez 12]. Disponível em: http://www.inmet.gov.br/

Kageyama PY, Gandara FB. Revegetação de áreas ciliares. In: Rodrigues RR, Leitão-Filho HF, editores. Matas ciliares: conservação e recuperação. São Paulo: Editora da USP/ FAPESP; 2000.

Kazanskii KS, Dubrovskii SA. Chemistry and physics of "agricultural" hydrogels. In: Abe A, Dus ek K, Kobayashi $\mathrm{S}$, editores. Polyelectrolytes hydrogels chromatographic materials. Heidelberg: Springer; 1992.

Klink CA, Machado RB. Conservation of the Brazilian Cerrado. Conservation Biology 2005; 19(3): 707-713. http:// dx.doi.org/10.1111/j.1523-1739.2005.00702.x.

Malaquias JV, Silva FAM, Evangelista BA. Precipitação pluviométrica em Planaltina, DF: análise de dados da estação principal da Embrapa Cerrados, 1974 a 2004. Planaltina: Embrapa Cerrados; 2010.

Mangold JM, Sheley RL. Effects of soil texture, watering frequency, and a hydrogel on the emergence and survival of coated and uncoated crested wheatgrass seeds. Ecological Research 2007; 25(1): 6-11. http://dx.doi.org/10.3368/ er.25.1.6.

Mews CL, Sousa JRL, Azevedo GTOS, Souza AM. Efeito do hidrogel e ureia na produção de mudas de Handroanthus ochraceus (Cham.) Mattos. Floresta e Ambiente 2015; 22(1): 107-116. http://dx.doi.org/10.1590/2179-8087.080814.

Monteiro MM. Efeito do hidrogel em plantios de mudas nativas do cerrado para recuperação de área degradada pela mineração no Distrito Federal [dissertação]. Brasília: Universidade de Brasília; 2014.

Moreira RA, Ramos JD, Cruz MCM, Villar L, Hafle OM. Efeito de doses de polímero hidroabsorvente no enraizamento de estacas de amoreira. Revista Agrarian 2011; 3(8): 133-139.

Oliveira RA, Rezende LS, Martinez MA, Miranda GV. Influência de um polímero hidroabsorvente sobre a retenção de água no solo. Revista Brasileira de Engenharia Agrícola e Ambiental 2004; 8(1): 160-163. http://dx.doi. org/10.1590/S1415-43662004000100023.

Rodrigues RR, Gandolfi S. Recomposição de florestas nativas: princípios gerais e subsídios para uma definição metodológica. Revista Brasileira de Horticultura Ornamental 2001; 2(1): 4-15.

Sano EE, Rosa R, Brito JLS, Ferreira LG. Mapeamento de cobertura vegetal do Bioma Cerrado. Planaltina: Embrapa Cerrados; 2008. Documentos, n. 190.

Sarvaš M. Effect of desiccation on the root system of Norway spruce (Picea abies [L.] KARST.) seedlings and a possibility of using hydrogel STOCKOSORB ${ }^{\star}$ for its protection. Journal of Forest Science 2003; 49(11): 531-536.

Sarvaš M, Pavlenda P, Takáčová E. Effect of hydrogel application on survival and growth of pine seedlings in reclamations. Journal of Forest Science 2007; 53(5): 204-209.

Sousa GTO, Azevedo GB, Sousa JRL, Mews CL, Souza AM. Incorporação de polímero hidroretentor no substrato de produção de mudas de Anadenanthera peregrina (L.) Speg. Enciclopédia Biosfera 2013; 9(16): 1270-1278.

Thomas DS. Hydrogel applied to the root plug of subtropical eucalypt seedlings halves transplant death following planting. Forest Ecology and Management 2008; 255(3-4): 1305-1314. http://dx.doi.org/10.1016/j.foreco.2007.10.035.

Venturoli F, Venturoli S, Borges JD, Castro DS, Sousa DM, Monteiro MM et al. Incremento de espécies arbóreas em plantio de recuperação de área degradada em solo de cerrado no Distrito Federal. Bioscience Journal 2013; 29(1): 143-151. 